\title{
Pleural Neutrophil-to-Lymphocyte Ratio May Be Associated With Early Disease Progression in Stage IV Non-small Cell Lung Cancer
}

\author{
JEONG UK LIM ${ }^{1}$, CHANG DONG YEO ${ }^{1}$, HYUNG WOO KIM ${ }^{1}$, HYE SEON KANG ${ }^{1}$, CHAN KWON PARK $^{1}$, \\ JU SANG KIM ${ }^{1}$, JIN WOO KIM ${ }^{1}$, SEUNG JOON KIM ${ }^{1,2}$ and SANG HAAK LEE ${ }^{1,2}$ \\ ${ }^{1}$ Division of Pulmonology and Critical Care Medicine, Department of Internal Medicine, \\ College of Medicine, The Catholic University of Korea, Seoul, Republic of Korea; \\ ${ }^{2}$ Cancer Research Institute, College of Medicine, The Catholic University of Korea, Seoul, Republic of Korea
}

\begin{abstract}
Background/Aim: While blood neutrophil-tolymphocyte ratio (NLR) has been associated with poor prognosis in NSCLC, however few studies have focused on pleural fluid white blood cell differential count. We conducted a retrospective multicenter cohort study to evaluate the predictive value of pleural NLR in non-small cell lung cancer (NSCLC) patients with malignant pleural effusion (MPE). Patients and Methods: From the multicenter lung cancer cohort, 134 epidermal growth factor receptor (EGFR) wild-type patients with NSCLC were selected for evaluation. Receiver operating characteristic (ROC) curve analysis was performed for pretreatment pleural NLR to determine the cut-off value for predicting disease progression within 100 days after the diagnosis. Results: The low-pleural NLR group showed significantly longer overall survival (OS) and progression free survival (PFS) compared to the high-pleural NLR group. After stratification using quartile cut-off values of pleural NLR, the correlation between risk of disease progression and pleural NLR was shown to be dose-dependent. The multivariate analysis on PFS showed that high-pleural NLR ( $p=0.004)$ was an independent predictor for shorter PFS with HR of 1.036 (1.011-1.061). Conclusion: Increased pleural NLR is predictive of early disease progression in EGFR mutation wild-type NSCLC patients with MPE.
\end{abstract}

This article is freely accessible online.

Correspondence to: Ju Sang Kim, MD, The Catholic University of Korea, Incheon St. Mary's Hospital, Bupyeong-gu, Bupyeong dong, Dongsu-ro 56, Incheon, Republic of Korea. Tel: +82 322805866 , Fax: +82 322805196, e-mail: kimjusang@catholic.ac.kr

Key Words: Non-small cell lung cancer, malignant pleural effusion, pleural neutrophil-to-lymphocyte ratio.
Non-small cell lung cancer (NSCLC) comprises $85 \%$ of all lung cancers (1). In $15 \%$ of lung cancer patients, malignant pleural effusion (MPE) occurs during the course of treatment (2) and MPE is a common complication for advanced NSCLC patients (3). Advanced NSCLC patients with MPE have a median survival time of less than 12 months (4). Furthermore, lung cancer patients with MPE complain of dyspnea, cough and chest pain, which lead to decreased quality of life (5).

It is well recognized that serum neutrophil-to-lymphocyte ratio (NLR) has shown proven prognostic value in various cancers $(6,7)$. In lung cancer, higher NLR is associated with a worse prognosis in stage IV NSCLC patients undergoing chemotherapy $(8,9)$. For NSCLC patients undergoing curative resection, higher NLR is associated with shorter overall survival (OS) (10-12). While serum NLR is proven to have a prognostic value in lung cancer, higher NLR suggests more active systemic inflammation which contributes to the pathogenesis of cancer progression (13). High NLR reflects neutrophilic inflammatory and relatively lymphopenic status; neutrophilic inflammation contributes to cancer progression, while lymphopenia reflects decreased antitumor activity $(14,15)$.

If the increased blood NLR is associated with systemic inflammation and lung cancer activity, it is also possible that pleural NLR also reflects local inflammatory status and tumor microenvironment of the pleural cavity and the nearby lung parenchyma. In turn, pleural NLR may have association with disease progression of lung cancer with MPE. If pleural NLR reflects increased inflammatory activity and has a predictability on early disease progression, it could be used as a quick and simple biomarker in NSCLC with MPE.

Furthermore, a reliable biomarker with predictive value on disease progression in patients with epidermal growth factor receptor (EGFR) wild-type NSCLC can be clinically important. Patients with EGFR mutations are eligible for targeted therapy 
and thus have a longer median survival compared to those without EGFR mutations (16); a readily available biomarker which can predict the treatment response can be useful in the relatively unfavorable EGFR wild-type NSCLC. The present study evaluated the predictive value of pretreatment pleural NLR on OS and PFS in patients with NSCLC with MPE.

\section{Patients and Methods}

Patient selection. Patients with stage IV NSCLC diagnosed between January 2012 and July 2017 were selected from records of six university hospitals: Seoul St. Mary's Hospital, Yeouido St. Mary's Hospital, Incheon St. Mary's Hospital, St. Paul's Hospital, Bucheon St. Mary's Hospital, and Uijeongbu St. Mary's Hospital. The inclusion criteria for enrollment were as follows (17): i) pleural effusion study was available before initiation of treatment; ii) patients had MPE which was proven either by pleural biopsy simultaneously that proved malignant or cytologically; iii) patients who had undergone 2 or more cycles of chemotherapy. Exclusion criteria were: i) patients who had small cell lung cancer (SCLC) and those who did not have primary lung cancer; ii) those who had significant infection which required antibiotics treatment; iii) those who had underlying hematological disease; iv) those who underwent curative resection or other surgeries involving pleural cavities.

In the present study, no patient underwent immune checkpoint inhibitor (ICI) therapy during the study period, because programmed cell death protein 1 (PD-1) and programmed death-ligand 1 (PDL1) tests, and ICIs including nivolumab and pembrolizumab were not a reimbursable expense for NSCLC treatment in Korean medical insurance system.

Clinical and laboratory data. Data including sex, age, histology, smoking status, tumor stages according to tumor-node-metastasis (TNM) criteria (AJCC criteria 2009), and Eastern Cooperative Oncology Group Performance Status Scale (ECOG PS) were evaluated for all study patients.

For all study subjects, both pleural and blood laboratory values were checked. A conventional cytologal examination was performed to identify malignant cells in pleural effusion fluid and check whether pleural biopsy tissue was malignant. Histological analysis was performed separately. After centrifuging at 2,500 rpm for about $10 \mathrm{~min}$, effusion fluid was examined for malignant cells. Two or more thin smears were prepared for the sediment. One was air-dried and stained with Leishman-Giemsa while the other was fixed in 95\% alcohol and stained with Papanicolaou stain.

Pretreatment complete blood count (CBC) and blood chemistry data were checked from the blood samples of the study patients. The difference in days between the dates of blood sampling and pleural fluid sampling was no more than three days.

Inflammatory markers. Serum NLR was calculated by dividing absolute neutrophil count by absolute lymphocyte count from pretreatment CBC, while pleural NLR was calculated by dividing absolute neutrophil count by absolute lymphocyte count from the pretreatment pleural fluid analysis. Platelet-to-lymphocyte ratio (PLR) was defined as a ratio of platelet count to absolute lymphocyte count, while lymphocyte-to-monocyte ratio (LMR) was defined as a ratio of absolute lymphocyte count to absolute monocyte count from pretreatment CBC.

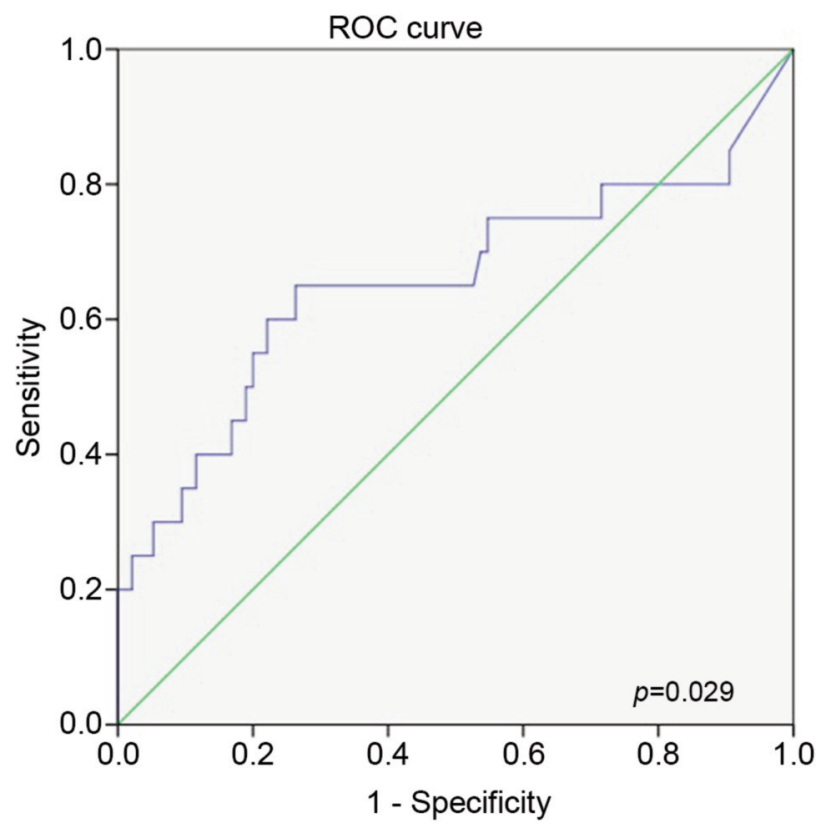

Figure 1. Receiver operator characteristics curve of pleural neutrophilto-lymphocyte ratio (NLR) for disease progression within 100 days after the diagnosis.

Overall survival and progression-free survival. Response Evaluation Criteria in Solid Tumors (RECIST) version 1.1 was used to evaluate response (18). After completing two cycles of treatment, computed tomography (CT) was performed. Responses were evaluated by the treating physicians and independent radiologists. OS was defined as the interval between the date of diagnosis and date of death. PFS was defined as the time from lung cancer diagnosis until disease progression after $1^{\text {st }}$ line treatment. If patients were dead or lost during the follow up period, they were censored, and the last observation was performed on October $10^{\text {th }}, 2018$.

Defining cut-off values. Receiver operating characteristic (ROC) curve was generated for pretreatment pleural NLR to determine the cut-off value for predicting progression-free survival for $<100$ days that yielded optimal sensitivity and specificity. The patients were then allocated to high/low groups based on the cut-off values.

Statistical analysis. All statistical analyses were performed using the Statistical Package for Social Sciences software program version 20.0 (SPSS Inc., Chicago, IL, USA). Continuous variables were shown as mean values. The Chi-squared test was performed to compare categorical variables between the groups. Continuous variables were compared between the groups using two-sided tests or Mann-Whitney test depending on normal distribution status. Using Kaplan-Meier analysis, survival curves were presented. Log-rank test was performed to assess the significance of difference in OS and PFS between the low and high-pleural NLR groups. Median OS and PFS were presented with 95\% confidence interval (CI). The Cox regression model was used to evaluate association with OS and PFS. Statistically significant variables from the univariate analysis were entered into the multivariate analysis using Cox proportional hazards regression model. Pleural NLR was included in the Cox proportional 
Lim et al: Pleural NLR in Malignant Pleural Effusion

Table I. Clinical characteristics of 134 EGFR wild-type NSCLC patients with malignant pleural effusion.

\begin{tabular}{|c|c|c|c|c|}
\hline Factor & Overall patients & High-pleural NLR & Low-pleural NLR & $p$-Value \\
\hline Number of patients & 134 & 50 & 84 & \\
\hline Median age, range & $69.0(32-96)$ & $68.0(41-96)$ & $71.0(32-85)$ & 0.345 \\
\hline Median OS (months) & $13.4(8.2-18.6)$ & $7.8(5.6-10.1)$ & $17.1(11.6-22.7)$ & 0.041 \\
\hline Median PFS (months) & $6.8(4.5-9.0)$ & $4.5(2.8-6.2)$ & $7.8(6.9-8.7)$ & 0.012 \\
\hline Gender & & & & 0.565 \\
\hline Male & $89(64.9)$ & $34(68.0)$ & $53(63.1)$ & \\
\hline Female & $47(35.1)$ & $16(32.0)$ & $31(36.9)$ & \\
\hline ECOG & & & & 0.182 \\
\hline 0 and 1 & $118(88.1)$ & $42(84.0)$ & $76(91.6)$ & \\
\hline$\geq 2$ & $15(11.2)$ & $8(16.0)$ & $7(8.4)$ & \\
\hline Histologic features & & & & $<0.001$ \\
\hline Adenocarcinoma & $113(84.3)$ & $35(70.0)$ & $78(92.9)$ & \\
\hline Squamous & $21(15.7)$ & $15(30.0)$ & $6(7.1)$ & \\
\hline Smoking & & & & 0.704 \\
\hline Never smoker & $64(47.8)$ & $23(46.0)$ & $41(49.4)$ & \\
\hline Ever smoker & $69(51.5)$ & $27(54.0)$ & $42(50.6)$ & \\
\hline M-factor & & & & 0.580 \\
\hline M1a/M1b & $72(53.7) / 53(39.5)$ & $29(58.0) / 17(34.0)$ & $43(51.2) / 36(42.9)$ & \\
\hline Treatment modality (1 ${ }^{\text {st }}$ line) & & & & 0.054 \\
\hline Supportive care only & $15(11.2)$ & $9(18.0)$ & $6(7.1)$ & \\
\hline Conventional chemotherapy & $119(88.8)$ & $41(82.0)$ & $78(92.9)$ & \\
\hline WBC count $\left(\times 10^{9} / 1\right)$ & $9518 \pm 4865$ & $11485 \pm 7052$ & $8347 \pm 2202$ & 0.003 \\
\hline Hemoglobin (g/dl) & $13.0 \pm 1.8$ & $12.4 \pm 1.7$ & $13.5 \pm 1.7$ & $<0.001$ \\
\hline Platelet (per/ul) & $308,900 \pm 82,365$ & $309,380 \pm 76,032$ & $308,610 \pm 86,359$ & 0.958 \\
\hline Albumin (g/dl) & $3.6 \pm 0.5$ & $3.4 \pm 0.5$ & $3.8 \pm 0.4$ & $<0.001$ \\
\hline $\mathrm{CRP}(\mathrm{mg} / \mathrm{l})$ & $2.5 \pm 4.2$ & $3.4 \pm 4.8$ & $1.9 \pm 3.8$ & 0.048 \\
\hline $\mathrm{CEA}(\mathrm{ng} / \mathrm{ml})$ & $70 \pm 156$ & $87.3 \pm 179.7$ & $59.3 \pm 142.0$ & 0.469 \\
\hline LDH (IU/l) & $503 \pm 234$ & $532 \pm 248$ & $486 \pm 226$ & 0.294 \\
\hline Serum NLR & $5.5 \pm 12.4$ & $9.1 \pm 20.0$ & $3.4 \pm 1.7$ & 0.049 \\
\hline Pleural NLR & $2.00 \pm 7.5$ & $5.26 \pm 11.79$ & $0.13 \pm 0.13$ & 0.004 \\
\hline PLR & $208 \pm 150$ & $249 \pm 218$ & $184 \pm 80$ & 0.047 \\
\hline LMR & $2.7 \pm 1.4$ & $2.2 \pm 1.1$ & $3.1 \pm 1.5$ & 0.001 \\
\hline
\end{tabular}

CI: Confidence interval; CEA: carcinoembryonic antigen; CRP: c-reactive protein; ECOG: Eastern Cooperative Oncology Group; HR: hazard ratio; LDH: lactate dehydrogenase; LMR: lymphocyte to monocyte ratio; OS: overall survival; PFS: progression-free survival; PLR: platelet to lymphocyte ratio; WBC: white blood cell.

hazard regression model as a continuous variable. The association between the outcomes and variable number of patients was presented as hazard ratio (HR) and $95 \%$ confidence interval (CI). A p-value less than 0.05 was considered statistically significant for all analyses.

Ethics statement. The study was approved by the Ethics Committee at each Center: Seoul St. Mary's Hospital, Yeouido St. Mary's Hospital, Incheon St. Mary's Hospital, St. Paul's Hospital, Bucheon St. Mary's Hospital, and Uijeongbu St. Mary's Hospital (XC17REDI0069U). Due to the retrospective nature of the data, the requirement for informed consent was waived. This study was conducted in accordance with the Declaration of Helsinki. All patient data in the present study were fully anonymized before evaluation.

\section{Results}

Predictability for disease progression. ROC curve analysis was performed to determine the cut-off value for the pretreatment pleural NLR that predicted disease progression within 100 days after the diagnosis. The calculated cut-off value for a pleural NLR was 0.536 [area under curve (AUC) $=0.656$; sensitivity $=0.650$; specificity $=0.737 ; p=0.029$ ] (Figure 1).

Using the cut-off calculated from the ROC curve analysis, the study patients were categorized into the high-pleural and low-pleural NLR groups. Among the overall patients, 50 patients were categorized into the high-pleural NLR group (pleural NLR >0.536), and 84 patients were categorized into the low-pleural NLR group (pleural NLR <0.536).

Patient characteristics. A total of 134 patients with NSCLC were found to have MPE that was proven cytologically or confirmed by pleural biopsy with malignant result. Baseline characteristics and other clinical parameters of these patients are shown in Table I. Median age of patients was 68.7 years (range $=32-96$ years), and a total of $89(64.9 \%)$ patients were male. The majority $(84.3 \%)$ of patients were diagnosed with 


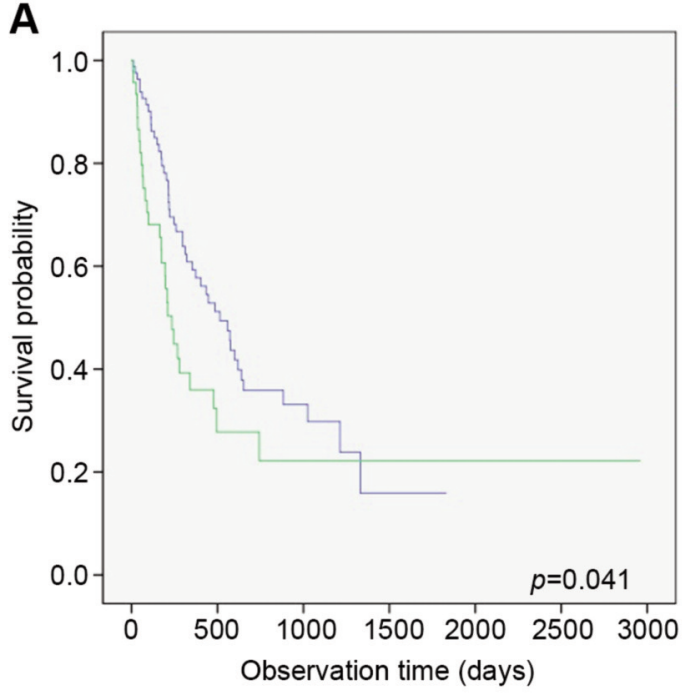

$\rightarrow P I N L R$ low group

PI NLR high group

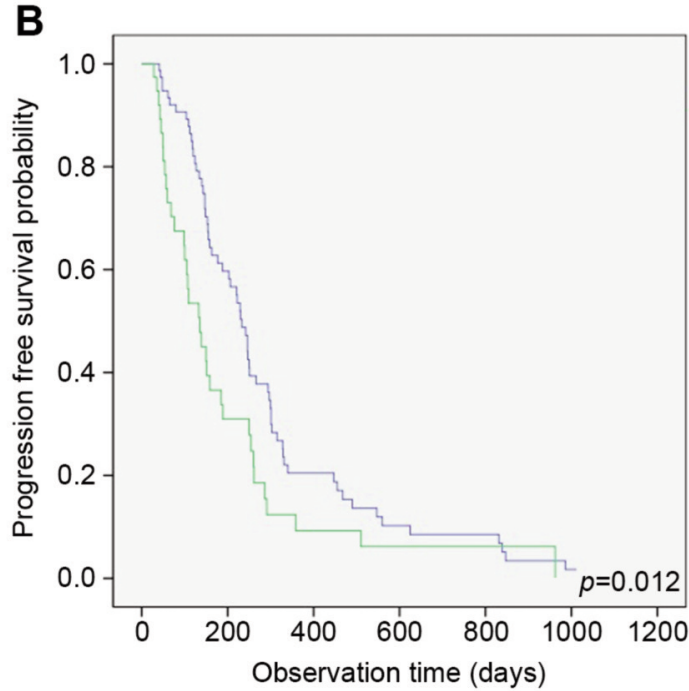

$\neg P I$ NLR low group PI NLR high group

Figure 2. Comparison of overall survival (A) and progression-free survival (B) between high-and low-pleural neutrophil-to-lymphocyte ratio (NLR) groups in non-small cell lung cancer (NSCLC) with malignant pleural effusion (MPE).

A

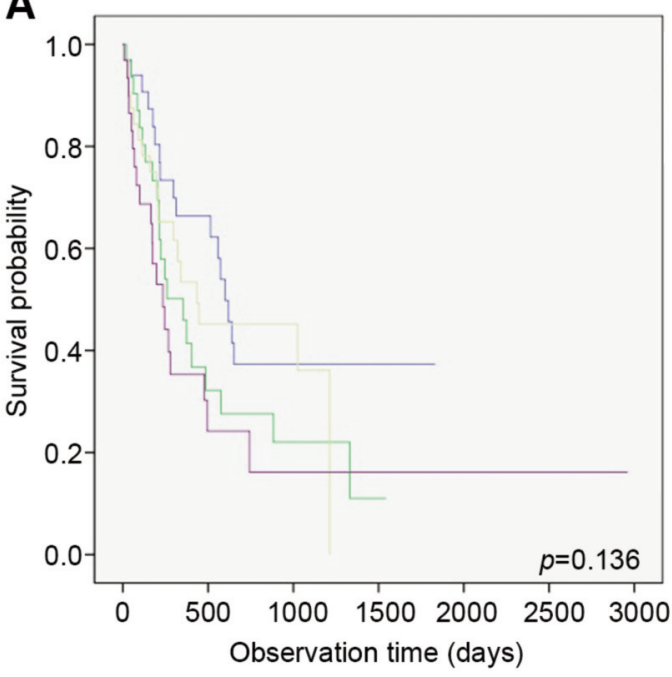

$\neg 0-25 \%$ quartile

$25-50 \%$ quartile

$50-75 \%$ quartile

$75-100 \%$ quartile
B

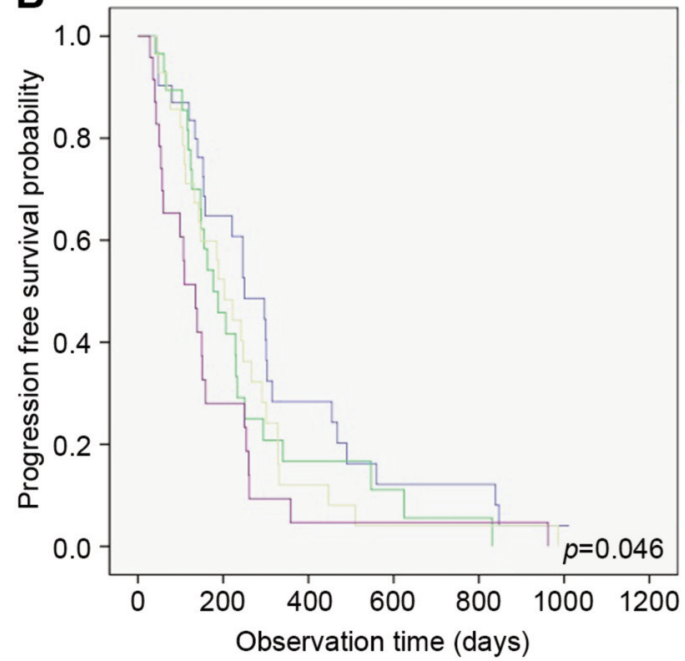

$\neg 0-25 \%$ quartile $25-50 \%$ quartile $50-75 \%$ quartile $\neg 75-100 \%$ quartile

Figure 3. Comparison of overall survival (A) and progression-free survival (B) between the four groups using quartile cut-off values of pretreatment pleural neutrophil-to-lymphocyte ratio (NLR).

adenocarcinoma. Most (88.1\%) patients had an ECOG PS of $0-1$. Among the study patients, $119(88.8 \%)$ underwent conventional systemic chemotherapy as first-line treatment, and $15(11.2 \%)$ patients received supportive care only.
Median OS was 13.4 months (8.2-18.6 months). PFS for $1^{\text {st }}$ line treatment was 6.8 months (4.5-9.0 months).

Median age in the low-pleural NLR group was higher than in the high-pleural NLR group. There was no statistically 
Table II. Univariate and multivariate analysis on $O S$ and PFS.

\begin{tabular}{|c|c|c|c|c|c|c|c|c|}
\hline \multirow[t]{3}{*}{ Characteristics } & \multicolumn{4}{|c|}{ OS } & \multicolumn{4}{|c|}{ PFS } \\
\hline & \multirow{2}{*}{$\begin{array}{l}\text { Univariate } \\
p \text {-Value }\end{array}$} & \multicolumn{3}{|c|}{ Multivariate } & \multirow{2}{*}{$\begin{array}{l}\text { Univariate } \\
p \text {-Value }\end{array}$} & \multicolumn{3}{|c|}{ Multivariate } \\
\hline & & $p$-Value & HR & $95 \% \mathrm{CI}$ & & $p$-Value & HR & $95 \% \mathrm{CI}$ \\
\hline Age & 0.009 & 0.126 & 1.025 & $0.993-1.057$ & 0.262 & 0.528 & 1.007 & $0.986-1.028$ \\
\hline Male & 0.019 & 0.342 & 1.355 & $0.725-2.532$ & 0.146 & 0.438 & 1.189 & $0.768-1.841$ \\
\hline Smoking status (ever/never) & 0.214 & - & - & - & 0.133 & - & - & - \\
\hline $\begin{array}{l}\text { Histology (adenocarcinoma/ } \\
\text { squamous cell) }\end{array}$ & 0.792 & - & - & - & 0.452 & - & - & - \\
\hline $\operatorname{ECOG}(0-1 / \geq 2)$ & 0.007 & 0.406 & 0.670 & $0.261-1.723$ & 0.125 & - & - & - \\
\hline $\begin{array}{l}\text { First line treatment }\left(1^{\text {st }} \text { line }\right) \\
\text { (conventional chemotherapy/ } \\
\text { supportive care) }\end{array}$ & $<0.001$ & $<0.001$ & 2.013 & $1.405-2.885$ & - & - & - & - \\
\hline Pleurodesis & 0.244 & - & - & - & 0.355 & - & - & - \\
\hline M stage (M1a/M1b) & 0.668 & - & - & - & 0.863 & - & - & - \\
\hline Hemoglobin, g/dl & 0.006 & 0.388 & 0.923 & $0.769-1.108$ & 0.213 & - & - & - \\
\hline Protein, g/dl & 0.036 & 0.715 & 1.105 & $0.648-1.883$ & 0.732 & - & - & - \\
\hline Albumin, g/dl & $<0.001$ & 0.073 & 0.419 & $0.162-1.084$ & 0.014 & 0.098 & 0.653 & $0.394-1.082$ \\
\hline LDH, IU/1 & 0.138 & - & - & - & 0.799 & - & - & - \\
\hline $\mathrm{CRP}, \mathrm{mg} / \mathrm{dl}$ & $<0.001$ & 0.007 & 1.115 & $1.031-1.207$ & 0.389 & - & - & - \\
\hline Pleural NLR & 0.117 & - & - & - & $<0.001$ & 0.004 & 1.036 & $1.011-1.061$ \\
\hline Serum NLR & $<0.001$ & 0.072 & 1.029 & $0.997-1.061$ & 0.160 & - & - & - \\
\hline PLR & $<0.001$ & 0.534 & 1.001 & $0.998-1.004$ & 0.019 & 0.090 & 1.002 & $1.000-1.005$ \\
\hline LMR & $<0.001$ & 0.417 & 0.897 & $0.691-1.166$ & 0.062 & - & - & - \\
\hline
\end{tabular}

CI: Confidence interval; CRP: c-reactive protein; ECOG: Eastern Cooperative Oncology Group; HR: hazard ratio; LDH: lactate dehydrogenase; LMR: lymphocyte to monocyte ratio; NLR: neutrophil-to-lymphocyte ratio; PLR: platelet-to-lymphocyte ratio; OS: overall survival; PFS: progression free survival.

significant difference in the proportion of male, ever smoker and patients who received active anticancer treatment between the two groups. The low-pleural NLR group showed a significantly higher proportion of adenocarcinoma when compared to the high-pleural NLR group (92.9\% vs. $70.0 \%$, $p<0.001)$.

The baseline laboratory test values of the study patients are shown in Table I. Mean white blood cell (WBC) count was 9,518. Mean levels of hemoglobin, platelet and albumin were $13.0 \mathrm{~g} / \mathrm{dl}, 308,900 / \mu \mathrm{l}$ and $3.6 \mathrm{~g} / \mathrm{dl}$, respectively. Mean creactive protein (CRP), carcinoembryonic antigen (CEA) and lactate dehydrogenase (LDH) were $2.5 \mathrm{mg} / 1,70 \mathrm{ng} / \mathrm{ml}$ and 503 IU/1, respectively. Mean serum NLR, pleural NLR and PLR were 5.5, 2.00 and 208, respectively. Mean LMR was 2.7.

Mean WBC count and CRP were significantly higher in the high-pleural NLR group than in the low-pleural NLR group (11485 vs. 8347, $p=0.003$, and $3.4 v s .1 .9, p=0.048$, respectively). Mean hemoglobin and albumin levels were significantly lower in the high-pleural NLR group than in the low-pleural NLR group (12.4 vs. 13.5, $p<0.001$, and $3.4 v s .3 .8, p<0.001)$. While, platelet count showed no significant difference, NLR, pleural NLR, and PLR were significantly higher in the high-pleural NLR group (9.1 vs. 3.4, $p=0.049,5.26$ vs. 0.13, $p=0.004$, and 249 vs. 184, $p=0.047$, respectively). LMR was lower in the high- pleural NLR group than in the low-pleural NLR group (2.2 vs. $3.1, p=0.001)$.

Overall survival and progression-free survival: Log-rank test. Between the two groups, OS and PFS were compared. The low-pleural NLR group showed a significantly longer median OS and PFS when compared to the high-pleural NLR group (17.1 vs. 7.8 months, $p=0.041$, and $7.8 v s .4 .5$ months, $p=0.012$, respectively) (Figure $2 \mathrm{~A}$ and $\mathrm{B}$ ).

After stratification using quartile cut-off values of pretreatment pleural NLR, Kaplan-Meier curve analysis was performed to compare OS and PFS between the four groups. Between the four categories, no significant difference in OS was found ( $p=0.136$ ) (Figure 3A). The graph from Kaplan Meier-curve analysis showed that as pleural NLR levels increase, the risk of disease progression increases as well $(p=0.046)$ (Figure 3B). As pleural NLR increases from $1^{\text {st }}$ quartile to $4^{\text {th }}$ quartile, median PFS changes as follows: 6.0, $9.8,10.0$, and 12.1 months, respectively.

Overall survival and progression-free survival: Coxregression model. Clinical parameters were entered into univariate analyses for association with OS and PFS: age, sex, smoking status, histology, ECOG, first line treatment, 
pleurodesis, M stage, hemoglobin, protein, albumin, LDH, CRP, pleural NLR, NLR, PLR and LMR (first-line treatment was not entered into the univariate analysis for PFS). The univariate analysis for association with OS showed that age, sex, ECOG, first-line treatment, hemoglobin, protein, albumin, CRP, NLR, PLR and LMR were significant factors. In the multivariate analysis for association with OS, first line treatment and CRP showed significant association (Table II).

Based on univariate analysis on association with PFS, albumin, pleural NLR and PLR were entered into multivariate analysis, along with age and sex. The multivariate analysis showed that only pleural NLR $(p=0.004)$ was an independent predictor for early disease progression, with HR=1.036 (1.000-1.005) (Table II).

\section{Discussion}

The present study showed that increased pleural NLR was an independent predictor for early disease progression in EGFR wild-type NSCLC patients with MPE. In addition, our study results showed that the correlation between PFS and pleural NLR was dose-dependent.

Higher NLR, which can be interpreted as more neutrophilic inflammation and lymphopenia state, was associated with poor survival in many cancers (19). Recent studies support that chronic inflammation in the tumor microenvironment promotes cancer progression and angiogenesis $(13,19)$. In depth, neutrophilic inflammation is associated with angiogenesis and tumor progression as well as poor outcomes in lung cancer $(14,20)$. In contrast, lymphocytes play an important role in anticancer activity (15).

Like NLR calculated from blood of NSCLC patients, NLR calculated from pleural fluid leukocyte analysis can give useful clinical information. MPE is basically lymphocytic, but MPE with relatively higher neutrophil percentage may reflect more active inflammation involving increased cytokine activity, and in turn, may have an association with cancer progression within and around the pleural cavity. A study on MPE of immunocompetent mice has shown that $\mathrm{T}$ lymphocytes and associated cytokines play significant roles in pathogenesis of MPE development (21). Increased levels of VEGF and related cytokines have been reported to have an association with formation of MPE (22).

In the present study, increased pleural NLR was independently associated with early disease progression of NSCLC with MPE. The disease progression included a meaningful increase of primary lung mass, a development of new mass in lung parenchyme, and a significant increase of MPE according to RECIST (18). As we hypothesized before, we assume that increased pretreatment inflammatory activity as was shown by relative neutrophilia, influenced cancer microenvironments of the pleural cavity and lung parenchyme. However, this hypothesis needs to be verified in a larger population with NSCLC and MPE before using this biomarker in clinical use.

The reason why pleural NLR was statistically significant in wild-type NSCLC patients should also be clarified. In a separate analysis involving our study cohort, pleural NLR was not significant in the EGFR mutation-positive NSCLC. We believe that the strong effect of targeted therapy on disease progression made predictability of pleural NLR not visible. Despite recent advances in treatment modalities for stage IV NSCLC patients, conventional chemotherapy remains the mainstay treatment in management of driver mutation-negative patients. The EGFR mutation wild-type NSCLC patients have a relatively poorer survival compared to EGFR mutation-positive NSCLC patients (16). With limited treatment options for first-line treatment and relatively poor prognosis, a prediction of disease progression is significant in this disease group. Therefore, a costeffective and more readily available biomarker can contribute to the management of NSCLC by making an easier prediction of early disease progression.

The present study has several limitations. First, median survival time of the patients was 13.4 months, which was significantly longer than the reported survival time of lung cancer with MPE (23). These could be due to the fact that patients with hematological disease and combined infections that would require antibiotics therapy were excluded. Second, pleural NLR was not measured serially and we could not evaluate whether pleural NLR demonstrates response after anticancer treatment. Finally, pleural NLR was not validated in the separate cohort, and this process should be performed in the large-sized population in the near future.

Despite its limitations, the present study showed that pleural NLR may assist in predicting prognosis in advanced NSCLC with MPE. This potential biomarker needs further affirmation for its clinical application, but its availability and cost-effectiveness deserve further attention from clinicians who manage lung cancer.

Pleural NLR was predictive of early disease progression in EGFR mutation wild-type NSCLC with MPE. Further studies involving a larger number of patients are necessary to clarify the correlation between pleural inflammation and cancer progression.

\section{Conflicts of Interest}

The Authors have no conflicts of interest to declare.

\section{Authors' Contributions}

JUL: Data analysis, visualization, writing, data collection; CDY: conception, data collection, critical revision of the article; HWK: data analysis; HSK, CKP, JWK, SJK: data collection; JSK: conception, data collection, critical revision of the article; SHL: data collection, project administration. 


\section{References}

1 Herbst RS, Heymach JV and Lippman SM: Lung cancer. N Engl J Med 359(13): 1367-1380, 2008. PMID: 18815398. DOI: 10.1056/NEJMra0802714

2 Sugiura S, Ando Y, Minami H, Ando M, Sakai S and Shimokata $\mathrm{K}$ : Prognostic value of pleural effusion in patients with nonsmall cell lung cancer. Clin Cancer Res 3(1): 47-50, 1997. PMID: 9815536.

3 Du N, Li X, Li F, Zhao H, Fan Z, Ma J, Fu Y and Kang H: Intrapleural combination therapy with bevacizumab and cisplatin for non-small cell lung cancermediated malignant pleural effusion. Oncol Rep 29(6): 2332-2340, 2013. PMID: 23525453. DOI: $10.3892 /$ or.2013.2349

4 Antunes G, Neville E, Duffy J, Ali N and Pleural Diseases Group SoCCBTS: Bts guidelines for the management of malignant pleural effusions. Thorax 58 Suppl 2: ii29-ii38, 2003. PMID: 12728148. DOI: 10.1136/thorax.58.suppl_2.ii29

5 Antony VB, Loddenkemper R, Astoul P, Boutin C, Goldstraw P, Hott J, Rodriguez Panadero F and Sahn SA: Management of malignant pleural effusions. Eur Respir J 18(2): 402-419, 2001. PMID: 11529302. DOI: 10.1183/09031936.01.00225601

6 Walsh SR, Cook EJ, Goulder F, Justin TA and Keeling NJ: Neutrophil-lymphocyte ratio as a prognostic factor in colorectal cancer. J Surg Oncol 91(3): 181-184, 2005. PMID: 16118772. DOI: $10.1002 /$ jso.20329

7 Gomez D, Morris-Stiff G, Toogood GJ, Lodge JP and Prasad KR: Impact of systemic inflammation on outcome following resection for intrahepatic cholangiocarcinoma. J Surg Oncol 97(6): 513518, 2008. PMID: 18335453. DOI: 10.1002/jso.21001

8 Cedres S, Torrejon D, Martinez A, Martinez P, Navarro A, Zamora E, Mulet-Margalef $\mathrm{N}$ and Felip E: Neutrophil to lymphocyte ratio (nlr) as an indicator of poor prognosis in stage iv non-small cell lung cancer. Clin Transl Oncol 14(11): 864869, 2012. PMID: 22855161. DOI: 10.1007/s12094-012-0872-5

9 Yao Y, Yuan D, Liu H, Gu X and Song Y: Pretreatment neutrophil to lymphocyte ratio is associated with response to therapy and prognosis of advanced non-small cell lung cancer patients treated with first-line platinum-based chemotherapy. Cancer Immunol Immunother 62(3): 471-479, 2013. PMID: 22986452. DOI: $10.1007 / \mathrm{s} 00262-012-1347-9$

10 Zhang H, Zhang L, Zhu K, Shi B, Yin Y, Zhu J, Yue D, Zhang $B$ and Wang C: Prognostic significance of combination of preoperative platelet count and neutrophil-lymphocyte ratio (cop-nlr) in patients with non-small cell lung cancer: Based on a large cohort study. PLoS One 10(5): e0126496, 2015. PMID: 25950176. DOI: 10.1371/journal.pone.0126496

11 Tomita M, Shimizu T, Ayabe T, Nakamura K and Onitsuka T: Elevated preoperative inflammatory markers based on neutrophil-to-lymphocyte ratio and c-reactive protein predict poor survival in resected non-small cell lung cancer. Anticancer Res 32(8): 3535-3538, 2012. PMID: 22843942.

12 Tomita M, Shimizu T, Ayabe T, Yonei A and Onitsuka T: Preoperative neutrophil to lymphocyte ratio as a prognostic predictor after curative resection for non-small cell lung cancer. Anticancer Res 31(9): 2995-2998, 2011. PMID: 21868550.

13 O'Callaghan DS, O'Donnell D, O'Connell F and O'Byrne KJ: The role of inflammation in the pathogenesis of non-small cell lung cancer. J Thorac Oncol 5(12): 2024-2036, 2010. PMID: 21155185. DOI: $10.1097 /$ jto.0b013e3181f387e4
14 Coussens LM and Werb Z: Inflammation and cancer. Nature 420(6917): 860-867, 2002. PMID: 12490959. DOI: 10.1038/ nature 01322

15 Lee TK, Horner RD, Silverman JF, Chen YH, Jenny C and Scarantino CW: Morphometric and morphologic evaluations in stage iii non-small cell lung cancers. Prognostic significance of quantitative assessment of infiltrating lymphoid cells. Cancer 63(2): 309-316, 1989. PMID: 2535956. DOI: 10.1002/10970142(19890115)63:2<309::aid-cncr2820630218>3.0.co;2-n

16 Kris MG, Johnson BE, Berry LD, Kwiatkowski DJ, Iafrate AJ, Wistuba, II, Varella-Garcia M, Franklin WA, Aronson SL, Su PF, Shyr Y, Camidge DR, Sequist LV, Glisson BS, Khuri FR, Garon EB, Pao W, Rudin C, Schiller J, Haura EB, Socinski M, Shirai K, Chen H, Giaccone G, Ladanyi M, Kugler K, Minna JD and Bunn PA: Using multiplexed assays of oncogenic drivers in lung cancers to select targeted drugs. JAMA 311(19): 1998-2006, 2014. PMID: 24846037. DOI: 10.1001/jama.2014.3741

17 Lim JU, Yeo CD, Kang HS, Park CK, Kim JS, Kim JW, Kim SJ and Lee SH: Prognostic value of platelet count and lymphocyte to monocyte ratio combination in stage iv non-small cell lung cancer with malignant pleural effusion. PLoS One 13(7): e0200341, 2018. PMID: 30005083. DOI: 10.1371/journal. pone. 0200341

18 Eisenhauer EA, Therasse P, Bogaerts J, Schwartz LH, Sargent D, Ford R, Dancey J, Arbuck S, Gwyther S, Mooney M, Rubinstein L, Shankar L, Dodd L, Kaplan R, Lacombe D and Verweij J: New response evaluation criteria in solid tumours: Revised recist guideline (version 1.1). Eur J Cancer 45(2): 228247, 2009. PMID: 19097774 . DOI: 10.1016/j.ejca.2008.10.026

19 Schreiber RD, Old LJ and Smyth MJ: Cancer immunoediting: Integrating immunity's roles in cancer suppression and promotion. Science 331(6024): 1565-1570, 2011. PMID: 21436444. DOI: $10.1126 /$ science. 1203486

20 Carus A, Gurney H, Gebski V, Harnett P, Hui R, Kefford R, Wilcken N, Ladekarl M, von der Maase H and Donskov F: Impact of baseline and nadir neutrophil index in non-small cell lung cancer and ovarian cancer patients: Assessment of chemotherapy for resolution of unfavourable neutrophilia. J Transl Med 11: 189, 2013. PMID: 23945200. DOI: 10.1186/ 1479-5876-11-189

21 Lin $\mathrm{H}$, Tong ZH, Xu QQ, Wu XZ, Wang XJ, Jin XG, Ma WL, Cheng X, Zhou Q and Shi HZ: Interplay of th1 and th 17 cells in murine models of malignant pleural effusion. Am J Respir Crit Care Med 189(6): 697-706, 2014. PMID: 24410406. DOI: 10.1164/rccm.201310-1776OC

22 Bradshaw M, Mansfield A and Peikert T: The role of vascular endothelial growth factor in the pathogenesis, diagnosis and treatment of malignant pleural effusion. Curr Oncol Rep 15(3): 207-216, 2013. PMID: 23568600 . DOI: 10.1007/s11912-0130315-7

23 Lee YS, Nam HS, Lim JH, Kim JS, Moon Y, Cho JH, Ryu JS, Kwak SM and Lee HL: Prognostic impact of a new score using neutrophil-to-lymphocyte ratios in the serum and malignant pleural effusion in lung cancer patients. BMC Cancer 17(1): 557, 2017. PMID: 28830378. DOI: 10.1186/s12885-017-3550-8

Received March 31, 2020

Revised April 9, 2020

Accepted April 16, 2020 